\title{
A Modified Mean Atomic Number Background Correction Using Off-Peak Interpolated Measurements
}

\author{
Omero Orlandini ${ }^{1}$, John Donovan ${ }^{2}$ and Benjamin Hanson ${ }^{3}$ \\ ${ }^{1}$ University of Texas at Austin, Austin, Texas, United States, ${ }^{2}$ University of Oregon, Eugene, Oregon, \\ United States, ${ }^{3}$ Corning Research and Development Corp., Corning, New York, United States
}

The Mean Atomic Number (MAN) technique for modelling and removing background X-ray radiation from characteristic X-ray signals significantly decreases analysis time by removing the need for background measurements on unknowns, while preserving excellent accuracy and even increasing precision on major, minor, and trace concentrations [1,2]. The calibration of the MAN background model requires the background radiation at the emission energies of interest to be measured directly in materials containing zero concentration of the element(s) of interest and across a range of mean atomic numbers likely to cover the primary standards and the unknown(s). Typical MAN standard materials consist of pure elements or simple binary compounds due to the need for high purity. Such standard materials rarely have the same mean atomic number as the unknown, meaning that the application of the background model to an unknown will likely rely on a range of mean atomic number between actual background measurements. Further, such high purity MAN standards tend to have more simple matrices than unknowns of interest, necessitating the use of more traditional standard suites in addition to the MAN standard suite, though as matrix corrections continue to improve in accuracy such matrix match considerations may no longer be necessary for primary standards.

We present a modified MAN background model calibration that utilizes the interpolated background intensities from a traditional off-peak background measurement set. The option to include these interpolated background measurements improves the MAN background modelling technique in several ways. Using off-peak interpolated values allows the use of any standard material, even those containing significant concentrations of the element of interest. This allows the analyst to easily populate the MAN calibration curve with many well-spaced background measurements, as well as increases the likelihood of a background measurement in a standard material that much more closely matches the mean atomic number and matrix of unknowns. This approach also simplifies the analysis of elements such as $\mathrm{O}$ which was frequently present at trace concentrations in traditional MAN standard suites. Lastly, this streamlines the standardization process by allowing on-peak measurements and MAN background measurements to all be collected from the same traditional standard suite the analyst might have used before the advent of MAN. We note that using off-peak interpolated background values exposes the study to all of the same potential pitfalls of off-peak background measurements as in point analyses, such as interferences on offpeak positions, curved backgrounds, and absorption edges, hence the original (on-peak) MAN method still provides the best possible accuracy for background determinations.

In this work, we demonstrate that careful use of off-peak background measurements on complex standards containing the element of interest may be used to calibrate a MAN background model which produces quantitative results approaching the accuracy, while maintaining similar precision to the original MAN. However, due to the possibility of undeclared trace contaminants in standard materials (see session A11) and the significant time savings of employing MAN background modeling in analyzing unknowns, we recommend a combination of interpolated and direct measurements as suits each particular laboratory's standard collection. 

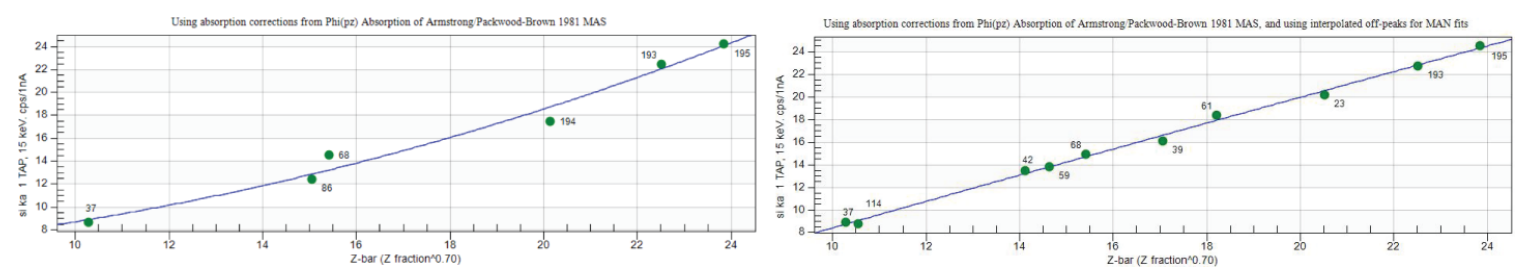

Figure 1. MAN fits for traditional MAN standards (left) and using interpolated background values (right) for $\mathrm{Si}$ collected at $20 \mathrm{nA}, 30$ s on both peak and background. Data plotted with 2-sigma error bars. Note that the interpolated method increases the number of usable standards from six to ten over the same mean atomic number range.
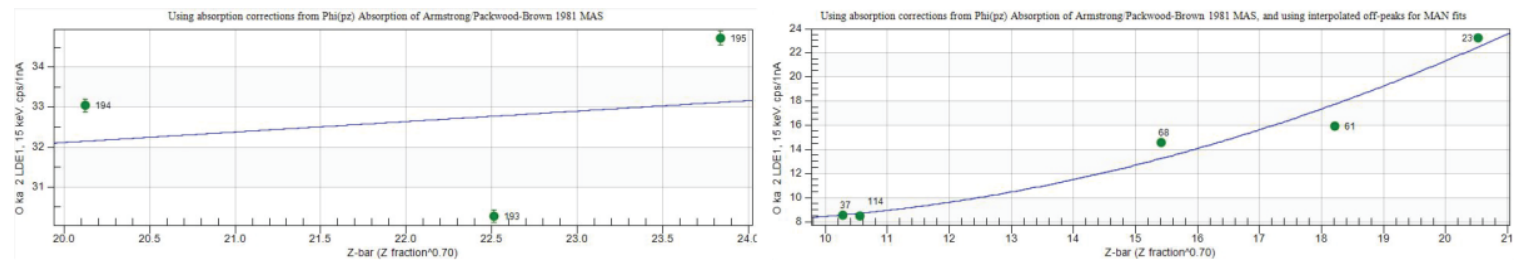

Figure 2. Same as figure 1, but for $O$. Note that the interpolated method increases the number of usable standards from three to five, and extends the calibrated range from 20-24 mean atomic number down to 10 (most geological silicates are 10-18).

\section{References}

[1] Donovan, JJ and Tingle, T, Journal of Microscopy and Microanalysis 2 (1996) p. 1.

[2] Donovan, JJ, Singer, JW and Armstrong, JT (2016) A New EPMA Method for Fast Trace Element Analysis in Simple Matrices", American Mineralogist, v101, p1839-1853 